\title{
Aprendizaje a través de proyecto en Diversidad e Identidades Étnicas en Asia Oriental
}

\section{Learning through challenges Project in Diversity and Ethnic Identities in East Asia}

ÁNGELES CASTAÑo MADROÑAL

ORCID: https://orcid.org/0000-0002-3617-8041

Universidad de Sevilla

Departamento de Antropología Social

acastamad@us.es

Fecha de recepción:

Fecha de aceptación:

DOI: http://dx.doi.org/10.12795/9788447221912.079

Pp.: 1812-1838 
En este trabajo se presenta un ciclo completo de mejora en el aula en la asignatura Diversidad e Identidades Étnicas en Asia Oriental, en el Grado en Estudios de Asia Oriental de la Universidad de Sevilla. Se trata de una metodología basada en el principio aprender-haciendo a través de un proyecto-reto de investigación bibliográfica. Los contenidos procedimentales de un trabajo académico cobran la misma relevancia que los propios contenidos teóricos y conceptuales del proyecto docente, promoviendo un rol pro-activo de los estudiantes en su formación y aprendizaje. Las habilidades tecnológicas de los estudiantes y la apertura digital de fuentes documentales, permiten una enseñanza basada en la indagación y el constructivismo educativo a partir de las producciones académicas que el conocimiento científico ha generado sobre la República Popular China y Japón desde la modernidad.

Palabras clave: diversidad e identidades étnicas en Asia Oriental, Grado en estudios de Asia Oriental, docencia universitaria, experimentación docente universitaria, aprendizaje a través de proyecto.

\section{Abstract}

This paper presents a complete cycle of improvement in the classroom in the subject Diversity and Ethnic Identities in East Asia, in the Degree in East Asian Studies of the University of Seville. It is a methodology based on the principle of learning-doing through a bibliographic research project-challenge. The procedural contents of an academic work acquire the same relevance as the theoretical and conceptual contents of the teaching project, promoting a pro-active role of students in their training and learning. The technological skills of the students and the digital opening of documentary sources, allow a teaching based on inquiry and educational constructivism, from the academic productions that scientific knowledge has generated about the Chinese People's Republic and Japan since modernity.

Key words: diversity and ethnic identities in East Asia, Degree in East Asian studies, university teaching, university teaching experimentation, project learning. 


\section{Antecedentes del proyecto de innovación}

El ciclo de mejora en el aula (CIMA) que voy a describir ha constado de 30 horas totales en una asignatura obligatoria cuatrimestral de 60 horas de clases y 6 créditos ECTS. Realizamos el CIMA desde el 25 de septiembre al 11 de noviembre de 2019. La asignatura es obligatoria de 30 curso del Grado de Estudios en Asia Oriental, y se imparte durante 2 sesiones de 2 horas semanales desde el 24 de septiembre de 2019 al 16 de enero de 2020. Este curso me he centrado en esta nueva asignatura de una titulación distinta que en proyectos anteriores: Diversidad e Identidades Étnicas en Asia Oriental del Grado mencionado de la Facultad de Filosofía. Es un título interuniversitario de la Universidad de Sevilla y la Universidad de Málaga. El motivo del cambio es la posibilidad de progresar a otras fases de innovación compartida mediante la colaboración y formación de equipo con el profesor Jesús Sanbernardino que también forma parte del profesorado de dicha titulación. El aislamiento de las innovaciones realizadas en el Grado de Antropología, al ser la única profesora de aquél grado en la REFID, impedía avanzar hacia nuevas experiencias. El aprendizaje en los ciclos sucesivos hasta abarcar la asignatura completa de antropología de la comunicación, que he mantenido en proyectos de innovación docente durante cuatro cursos académicos, me permite abarcar esta asignatura de modo completo haciendo transferencia de cuanto sea posible de todo lo aprendido y experimentado. La naturaleza de esta asignatura y este grado, requiere evidentemente una importante adaptación, pero considero que me encuentro en disposición de poder acometerla en su conjunto.

Se trata de una asignatura con un número mucho mayor de alumnado, concretamente este curso 2019/20 se encuentran matriculados 63 alumnos. Hay dificultades para experimentar a través de innovación educativa basada en conocimiento del medio ya que se abordan problemáticas 
específicas contextuales de la RPC y Japón, dificiles de llevar a la experimentación en Sevilla mediante principios de conocimiento experiencial. Los estudiantes no adquieren conocimientos previos en la titulación, ni en asignaturas del propio nivel de tercer curso, de los contenidos focales de la asignatura; es decir, se enfrentan por primera vez a las temáticas y a la conceptualización académica especializada que se emplea en el tratamiento de las minorías étnicas y la diversidad cultural.

Debido a esta particularidad, no he podido clonar los procesos llevados a cabo en años anteriores en los proyectos de innovación en el grado de antropología social. Pero he articulado un proceso basado en la participación de los estudiantes en su propio proceso de aprendizaje, las nuevas tecnologías audiovisuales y digitales, y el desarrollo creativo de trabajos académicos a través de un aprendizaje basado en retos para construir los pilares de esta experimentación docente universitaria a través de proyectos desarrollados por los estudiantes desde sus intereses y motivaciones.

\section{Diseñando un CIMA para aprender sobre etnias y diversidad en Asia Oriental}

La asignatura Diversidad e Identidades Étnicas en Asia Oriental debe centrarse en los recorridos formativos del Grado, que tiene mención en China y Japón. Por ello, los temas se centran en la cuestión de la diversidad en estos dos estados-nación modernos. Los estudiantes que se gradúan en una sola mención pueden centrar sus trabajos en su especialidad, pero todo el alumnado recibe conocimientos de ambos Estados de modo que adquieren una perspectiva contrastada, comparada y sistémica, con referencias también al modo en que las cuestiones se presentan en otras regiones del mundo. Una preocupación que sostengo en la asignatura es concienciar y sensibilizar 
sobre el sesgo orientalista y el esencialismo cultural que caracteriza una particular mirada exotizadora de los países orientales o asiáticos.

La asignatura tiene 5 temas:

- Tema1. Introducción a los conceptos centrales en la Antropología Social y CCSS para atender y comprender las cuestiones de diversidad e identidad étnica: cultura, diversidad cultural, multiculturalidad, interculturalidad, etnia, etnicidad, etnocentrismo, nación, nacionalismo, racismo, genocidio y etnocidio.

- Tema 2. Los estados-nación y las diversidades internas: El proceso chino y el proceso japonés. De la Modernidad a la Globalización. Procesos en los nacionalismos de Estado de China y Japón en la historia reciente. Cultura y nacionalismo estatal.

- Tema 3. Aproximación a la diversidad etnolingüística en la RPCh. El concepto cultura en la tradición Occidental vs. el concepto tradición en China. Minorías en la RPCh.

- Tema 4. Aproximación a la diversidad cultural en Japón. Minorías en Japón. La gestión de la diversidad cultural en el imaginario nacional de Japón a partir de la IIGM.

- Tema 5. Las etnicidades emergentes en la globalización en RPCh y Japón. Etnicidades emergentes en Asia. Crisis medioambiental sistémica y conflictos étnicos. La paradoja de la preservación cultural en el globalismo: el dilema entre universalismo y diversidad cultural humana. Tendencias y riesgos. 


\section{Mapa de contenidos}



Figura 1. Contenidos de Diversidad y Minorías en China y Japón.

El sistema de evaluación que he establecido permite que los estudiantes muestren los contenidos actitudinales, procedimentales y conceptuales adquiridos no sólo en esta asignatura, sino respecto a contenidos transversales con otras asignaturas de historia medieval, moderna y contemporánea, y filosofía de Asia Oriental. Los estudiantes deben entregar un producto creativo original basado en el desarrollo de un proyecto-reto: aplicar a un caso de estudio de minoría étnica o diversidad sociocultural en la RPC o Japón, seleccionado por ellos, las teorías generales de la ciencias sociales y la antropología social, e investigar sobre la producción científica directamente relacionada con el caso para enfocar las especificidades del grupo y el proceso en que se encuentran. Se trata de un trabajo colaborativo en coautoría entre pares, basado en investigación bibliográfica. Exponen a sus compañeros los resultados y entregan un trabajo escrito siguiendo las normas de formato y composición que les he elaborado. De este modo, adquieren a través del "aprender haciendo" los conocimientos procedimentales específicos de gestión 
académica de fuentes científicas. Dicho trabajo supone un eje transversal a través del cuál van articulando y aplicando los contenidos temáticos, y supone una experiencia de iniciación a la investigación bibliográfica y gestión de fuentes científicas: se facilitan lecturas obligatorias y recomendadas, un modelo de ficha bibliográfica que permite aprender a gestionar y explotar fuentes escritas antes de su volcado a una base de datos personal (la fase de volcado no se realiza), una bibliografía general amplia, y unas normas de elaboración de trabajos, estructuración y formato de citación de fuentes científica que se ensaya y explica en clases. Además, se imparten algunas sesiones sobre búsqueda bibliográfica en bases de datos accesibles desde la Universidad de Sevilla. En definitiva experimentan una iniciación a la investigación bibliográfica para trabajo escrito de formato artículo, útil, necesaria y transferible antes de que cursen la asignatura de Trabajo Fin de Grado en 4으 curso.

Los contenidos fundamentales para dicho proyecto-reto (T.R.) quedan identificados en el mapa de contenidos (figura 1). En sí mismo es una guía que puede ser útil para orientarles a lo largo del curso. Por ello, este año opté por compartirles y explicarles el mapa de contenidos, para orientarles en el estudio, la investigación y en la selección de los estudios de caso. Los contenidos conceptuales (C) forman parte de la unidad temática primera y se encuentran luego transversalmente en el resto de temas. Los contenidos procedimentales $(P)$ tienen que ver con el manejo de los conceptos y la identificación de problemáticas a través de las lecturas obligatorias que se facilitan en la plataforma de enseñanza virtual de la asignatura, que tengo construida en Moodle. Los contenidos actitudinales (A) tiene que ver con aquellas cuestiones que los estudiantes deberán comprender, interpretar y ponderar para trasladarlas a los debates en el aula, a la reflexión crítica personal, y a una aplicación reflexiva en sus estudios de caso. 


\section{Diseño metodológico del CIMA y secuencia de actividades.}

\section{Modelo metodológico ideal}

El modelo metodológico ideal permitiría abordar los contenidos con un aprendizaje a partir del conocimiento del medio, participativo, en equipos de trabajo, que permita desarrollar un aprendizaje y transferencia entre pares, y basado en retos de investigación y resolución de problemas para ir del medio al desarrollo teórico. Debería basarse en un aprender-haciendo (Rodríguez y Ramírez, 2014) que implique al estudiante plenamente en la construcción de su conocimiento, permitiéndole a través de lo experiencial desarrollar un sentido crítico ante la realidad que le rodea y una disposición a desaprender para aprender cosas nuevas de otros modos posibles.

Sin embargo, la asignatura se hace dificil para los estudiantes por centrarse en contenidos que no vuelven a afrontar en su grado, y por el desconocimiento de la antropología y de sus métodos de trabajo. Al margen de que la ubicuidad en Sevilla no permite un acercamiento a las minorías asiáticas tan lejanas epistemológica y geográficamente hablando. Más bien, la propia contextualidad local, promueve una percepción de homogeneidad de los migrantes asiáticos en la ciudad, cuya distribución diseminada en barrios y en su área metropolitana dificulta mucho un acercamiento como colectivos específicos. Por otro lado, las problemáticas estudiadas se comprenden en el contexto de sus respectivos lugares originales, ya que las diásporas imprimen otras variables muy complejas de considerar cuando ni siquiera tienen las bases para esta asignatura. 
Los límites temporales y la necesidad de partir de sus modelos mentales, que pueden estar bastante establecidos por la cantidad de contenidos de historia, de economía, de comunicación, de filosofia, de geografia, junto a sus propios imaginarios mediáticos sobre Asia que los atraen hacia este grado, obligan a ceñirse a un modelo más real y posible, de modo que al finalizar las 15 semanas de clases, se haya abierto en sus aprendizaje una serie de ventanas hacia diversas cuestiones y conocimientos que podrían retomar por interés particular más adelante, como en su TFG, o titulaciones posteriores. También considero necesario que adquieran conocimientos que vayan más allá de la propia asignatura, que desarrollen a su vez aprendizajes no solo de contenidos sino de procedimientos que puedan aplicar en otros ámbitos de profesionalización en el futuro.

\section{Modelo metodológico posible}

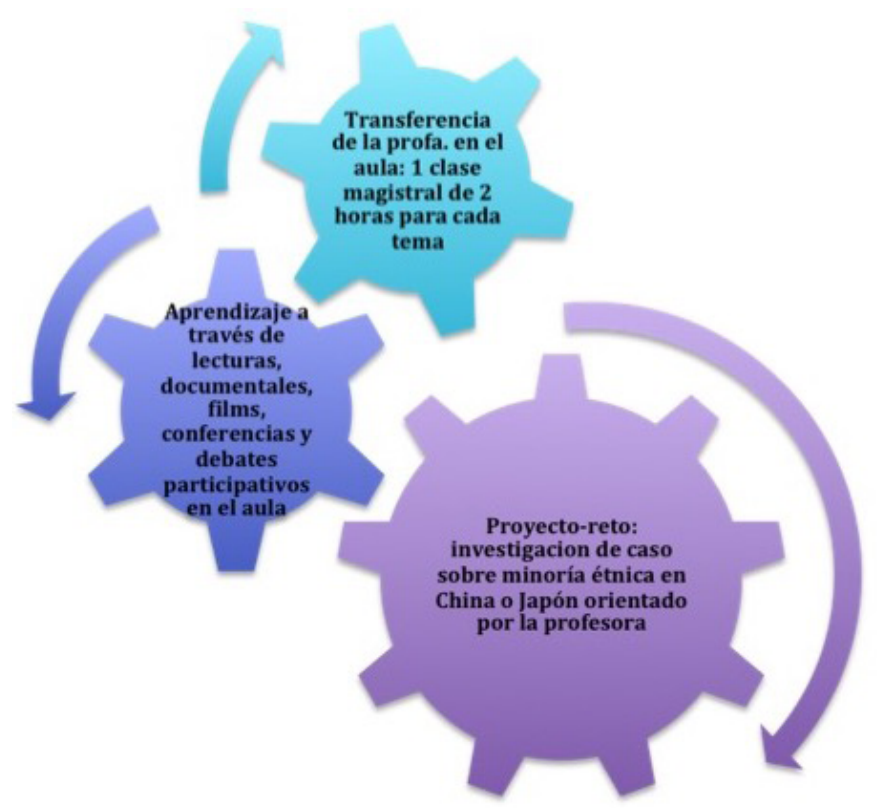

Figura 2. Modelo Metodológico Posible

Jornadas de Formación e Innovación Docente del Profesorado I № 2 (2019) Esta obra se distribuye con la licencia Creative Commons Reconocimiento-NoComercial-SinObraDerivada 
El modelo posible parte de un proyecto basado en retos realizado en coautoría entre pares desde que empieza el curso hasta que termina, y que deben entregar por escrito el día del examen oficial de la asignatura. Este proyecto se explica en clase el primer día del curso, así como las herramientas para poder desarrollarlo, y el procedimiento para acceder a la plataforma virtual de enseñanza donde se encuentran, a libre disposición todos, los contenidos y recursos docentes y de aprendizaje que pueden manejar durante el curso. El proyecto consta de una serie de herramientas instrumentales de manejo de información y fuentes científicas que ha elaborado la profesora para facilitar el aprendizaje de procesos de gestión de información junto al de contenidos de la asignatura: fichas bibliográficas de explotación de fuentes, instrucciones de formato y estructura para realizar un trabajo escrito de corte académico y guía de búsqueda de fuentes en bases de datos.

El proyecto-retos consta de cuatro fases:

1) Delimitación de un caso para su posible estudio mediante investigación bibliográfica (se orienta en el aula por la profesora a partir de las cuestiones de interés de los propios estudiantes).

2) Investigación de fuentes científicas procedentes de bases de datos accesibles. Aprendizaje de estos procedimientos y sus herramientas al alcance de todos (apoyo en la guía elaborada por la profesora, las explicaciones en el aula y las tutorías).

3) Estructuración de las ideas y cuestiones centrales del trabajo para transferencia en el aula: identificación de las ideas principales y secundarias y de las dificultades y desafios encontrados en la elaboración (apoyo en el modelo de ficha bibliográfica)

4) Redacción del trabajo y entrega al final del curso (seguimiento de las normas e instrucciones para su elaboración diseñadas por la profesora). 


\section{Secuencia de actividades}

Por otro lado, y de acuerdo a los estudios de caso, a lo largo del cuatrimestre se ordenan en cada bloque temático del mapa de contenidos al menos 1 clase práctica participativa en el aula que con ayuda de nuevas tecnologías y recursos audiovisuales permita traer a colación las problemáticas que están investigando y que ayude a los equipos de pares a identificar asuntos a atender, a desarrollar una perspectiva comparada con las informaciones que afectan a los distintos casos, y a debatir críticamente a partir de las fuentes que consultan y de las opiniones y debates participativos. La clase práctica se ayuda de films, documentales especializados, y videos sobre diversidad cultural en China y Japón, exposiciones y/o conferencias que se realicen en la universidad o que haya ocasión de realizar para la asignatura y comentarios de lecturas seleccionadas. La clase práctica ocupa 2 sesiones de 4 horas y en total son 5 una por cada tema: una completa para ver el documental/conferencia/lectura y otra para el debate y elaboración de conclusiones participativas. Esta clase práctica es anterior a 1 clase magistral de 2 horas también ubicada en cada bloque. De este modo, se va del debate y la clase práctica a los contenidos teóricos. En total suponen 7-8 semanas del curso. De las semanas restantes, 5 se ocupan en trabajo en el aula, y las sesiones se gestionan en función de las necesidades que tienen los estudiantes; es decir, a demanda colectiva para resolver dudas y solventar las dificultades y retos que se les va presentando conforme ahondan en sus trabajos. Esto se hace compartiendo la experiencia a nivel colectivo y por transferencia entre los equipos de pares, o de la profesora si se hace necesario. Las últimas 2 se dedican a un ejercicio de exposición oral de $15^{\prime}$ por equipo, sobre la experiencia que han tenido en la elaboración de su proyecto y sus hallazgos destacados. El último día una sesión de 1 hora de (auto) evaluación del aprendizaje y la docencia completa. 
Junto a estas actividades docentes, se han creado unos instrumentos de innovación destinados a identificar los mapas mentales de partida de los estudiantes, y su progreso en el aprendizaje y adopción de nuevos conocimientos. De esta forma se ha elaborado unos cuestionarios de preevaluación y posevaluación que se realizan antes y después de cada bloque de contenidos.

También se ha elaborado un cuestionario de evaluación del método de enseñanza llevado a cabo por la profesora que se pasa al final del CIMA. Y se ha incorporado 1 sesión de 2 horas de observación entre pares que realizamos CIMA completo y somos miembros de la REFID. De este modo, la reflexión a través de la observación compartida y cruzada de las prácticas ayudará a mejorar y modificar la comunicación en el aula. Esta observación entre pares se realizará a la mitad del CIMA, de modo que sea posible realizar cambios para el resto del ciclo. Se realizará en intercambio con el profesor Jesús Sanbernardino y está prevista en mi caso para el día 16/10/2019. Esta observación se recogerá en un diario compartido de la actividad para la reflexión conjunta posterior.

Tabla 1. Secuencia de actividades del ciclo de mejora

\section{Objetivo (0) - Instrumento Innovación (I)}

$\begin{array}{ll} & \text { Explicar } \\ \text { Bienvenida } & \text { Actividades del } \\ \text { CIMA }\end{array}$

Mapas mentales

de partida (O) - cuestionario tema $1(\mathrm{I})$

\section{Actividad}

CIMA

Cuestionario preevaluación (30-40')
Contenidos

Temporalidad

1 o y 2 o día

(D)/semana

$1(\mathrm{~S})$

Tema 1 - cuestionariopreevaluación sobre aspectos centrales de los contenidos y los problemas-retos en el marco de la asignatura. 


\section{Planteamiento}

Problema General Asignatura: debate participativo y video de $7^{\prime}($ I)

Clase Magistral (CM) - apoyo $\mathrm{TIC}(\mathrm{I})$

Clase Participativa (CP) - (O) powerpoint/construcción de la sesión desde conocimientos alumnos/ debates (I)

$\mathrm{CP}$ - (O) manejo herramientas de trabajo en Moodle: recursos didácticos prospectivos (I)
Debate participativo y reflexión.

\section{Conceptos}

Claves Recurso Visual

Conceptos

Claves Debate conclusiones participativas
Tema 1.

Homo Sapiens

productor de cultura.

Hecho social/biológico/ 3 D/S 2

histórico. ¿Quienes

somos?: cultura e

identidad

Tema 1

La cultura desde

la antropología

social y cultural. Sus

características. La

$4 \mathrm{D} / \mathrm{S} 2$

diversidad como bios

ecológico de la especie.

Casos y ejemplos

comparados.

Tema 1

Etnia/ nación/

nacionalismo/ minorías

étnica. La cuestión

étnica como problema

del estado-nación

(Stavenghagen/Barth).

Reflexión comparada

con la RPC y Japón.

Transversal

La prospección de

Herramientas procedimentales para Trabajo Retos entre pares - Moodle fuentes en un diseño

de proyecto. Testado

sobre estado de la

prospección para los

TR. Recogida de casos

seleccionados y datos

de los grupos.

Tema 1

Multiculturalidad/

interculturalidad/

CP - (O) - powerpoint/ construcción de la sesión desde conocimientos alumnos/debates (I)

Conceptos

Claves - Debate

- conclusiones participativas globalización/ glocalización/racismo/ genocidio/etnocidio/ DDHH: consecuencias e instrumentos que nos hemos dado.
$6 \mathrm{D} / \mathrm{S} 3$

$7 \mathrm{D} / \mathrm{S} 4$ 
Mapas

mentales (O).

cuestionario

tema $1(\mathrm{I})$

$\mathrm{CP}(\mathrm{O})$

debate

participativo

y video de

$7^{\prime}(\mathrm{I})$

Mapas mentales

de partida (O) - cuestionario tema 2-3 (I)

Observación entre

Pares
Observación

entre

Pares (O) (I)
Cuestionario de posevaluación $\left(30^{\prime}-40^{\prime}\right)$
Tema 1

Cuestionario-

posevaluación.

Observación

$8 \mathrm{D} / \mathrm{S} 4$

comunicación en el

aula y actitud ante el

ejercicio

\section{Tema 1}

Elaboración conjunta

Reflexión final/ conclusiones Tema 1.

conclusiones

Cual es la situación

tema 1.

Resolución en la globalización?.

dudas concep.

Diario

Compartido de Observaciones entre pares

Existen en China y Japón 8 D/S 4

casos identificables

a los conceptos

estudiados?. Ver

comparativamente a

otros lugares del mundo

con ejemplos.

Tema 2-3 - cuestionario

de preevaluación sobre

Cuestionario de

preevaluación

(30-40')

comprensión aspectos

centrales que articulan

los contenidos y los

$9 \mathrm{D} / \mathrm{S} 5$

problemas-retos para

la comprensión estado-

etnias y el caso RPC

Tema 2 - comunidad

imaginada y estado-

nación. Genealogía

Debate

moderna de los

Planteamiento Problema

General del Bloque Temático

2-3-4 (0). Debate participativo

a partir de lectura previa (I)

participativo

y reflexión-

conclusiones

participativas estados-nación.

Un modelo global

poscolonial. Diversidad

$9 \mathrm{D} / \mathrm{S} 5$

cultural y étnica y

homogeneización

nacional (Anderson/

Tanaka/Morris/

Yaqing/W.Hui). 
CP (O) - Tema 2- debate participativo a partir de lectura previa (I)

CP (O) - Tema 2- debate participativo a partir de lectura previa (I)

CM apoyo TIC (I)

CP (O) - Tema 3- DVD o Conferencia (I)

CP (O) - Tema 3- debate participativo a partir de DVD o Conferencia (I)
Recursos

Audiovisuales

Debate

participativo-

Conclusiones

Contenidos

teóricos

Recurso

audiovisual /

conferencia

invitación

Debate

participativoConclusiones
Tema 2 - comunidad imaginada de sinitud. Sinocentrismo. Del imperio al estadonación s. XX RPC.

Nuevas bases

$10 \mathrm{D} / \mathrm{S} 5$

culturales de un nuevo

estado: putonghua

y otros procesos

cohesionadores

(aplicación Anderson)

Tema 2 - comunidad imaginada de niponidad. El estadonación pos-IIGM. Imagen exterior y recomposición imagen interna de niponidad (aplicación Anderson).

Tema 2 - análisis comparado del proceso chino y japonés.

Adaptaciones del imaginario nacional: Tian-Xia/hogar-mundo. Kokutai/nihonjinron. Naciones, modernidad y colonialidad.

Tema 3 - DVD o conferencia relacionado con diversidades

$13 \mathrm{D} / \mathrm{S} 7$ internas en la RPC.

Tema 3 - La diversidad etnolingüística.

Identificación de grupos. Representaciones sobre

$14 \mathrm{D} / \mathrm{S} 7$ las minorías. Influencias del modelo estalinista. 
Evolución

Mapas mentales (0) -

cuestionario tema 2-3 (I)

EVALUACIÓN DE LA

DOCENCIA Y EL CIMA (0)
Cuestionario posevaluación $\left(30^{\prime}-40^{\prime}\right)$
Tema 2-3 - cuestionarioposevaluación

comprensión aspectos

centrales articuladores

de contenidos y

problemas-retos

para comprensión

estadonación-etnias en

caso RPC

\section{FIN DE CIMA -}

cuesrtionario de

evaluación de docencia.

Diálogo sobre el

proceso experimentado

con los estudiantes.

\section{Desarrollo y evaluación del CIMA.}

He promovido que los estudiantes realicen trabajos que les permitan comprender el mundo en que viven y la realidad contemporánea que afecta al caso que estén estudiando en una zona del planeta que estudian en la titulación de grado, pero de la que no tienen conocimiento directo. Los casos de estudio seleccionados para sus investigaciones por los estudiantes se recogen en la siguiente tabla, y abarcan grupos étnicos y/o socioculturales diversos (uigur, bai, hui, yi, nashi, ainu, zainichi, entre otros) atravesados por problemáticas como conflictos políticos, segregación étnica, folklorización y turistificación, desaparición de lenguas vernáculas, etc.:

Tabla 2. Casos de estudio seleccionados por los estudiantes

EQUIPO

(1) M.G.P.\& M.M.B.

(2) J.F.G.\&A.N.B.

(3) M.L.V.\&A.P.R

\section{TÍTULO PRELIMINAR O CASO}

Las minorías musulmanas en China: dinámicas actuales ente uigur y hui

La etnia china yi y el caso de la ciudad de Chuxiong

La etnia ainu en Japón

Jornadas de Formación e Innovación Docente del Profesorado | № 2 (2019) Esta obra se distribuye con la licencia Creative Commons Reconocimiento-NoComercial-SinObraDerivada Internacional (CC BY-NC-ND 4.0.) 
(4) A.A.R.\&E.C.G. Diversidad étnica en Xinyiang y política estatal.

(5) D.E.S.\&B.R.Y. Islas Ryükyü. Etnohistoria y actualidad.

(6) I.G.A.\& J.Y.M. Aproximación a la diáspora china en España.

(7) M.M.R.\&L.S.R. Recreación histórica activa, género e identidad bai en China.

(8) I.G.M.\&M.P.P. Los conflictos con la etnia uigur en China

(9) M.C.Q.\&T.G.M. La situación actual de los zainichi en Japón

(10) P.F.F.\&C.H.T. Turismo étnico. La folklorización de la cultura ainu

(11) V.P.P.\&.C.G. Evolución de las Geishas como transformación de género

(12) C.H.P.\&F.M.O. La problemática ainu en Japón.

(13) A.M.P.\&P.D.M. La problemática tibetana: evolución y situación actual

(14) B.Z.R.\&E.M.S. Asiacentrismo en China: lógicas económicas y culturales

(15) D.J.F.\&A.Y.C. Nakhi/nashi. Una minoría étnica en China

En función del caso de estudio los contenidos quedan delimitados en mayor o menor grado por las cuestiones que se plantean en la ilustración de los contenidos y problemas-retos que pueden ser identificados por los estudiantes (figura 1). Si bien, los conceptos comprendidos en el vértice de la ilustración son fundamentales para una correcta interpretación de los textos que deben manejar, por ser básicos en el campo especializado. La tabla que reproducimos registra los datos y casos seleccionados a partir de la primera prospección indagatoria que realizan. Ya en sí muestran una serie de aspectos a considerar en los mapas mentales de los estudiantes: identifican una serie de grupos como etnias a partir de la información digital a la que acceden $(1,2,3,7,8,9,10,12,15)$; aparecen territorios identificados como étnicos $(2,4,5,13)$; identifican como objeto de interés procesos que experimentan los grupos étnicos en la globalización $(6,10)$; tienen interés en procesos civilizatorios globales (11, 14); tienen cierto sesgo ideológico (imaginario mediático del mundo) al representar como "problemática" ciertas etnias o territorios etnizados construidos por confrontación $(8,12,13)$. 


\section{Los modelos mentales de los estudiantes}

Los modelos mentales previos de los estudiantes son fundamentales para orientar los contenidos en cada tema, hacer hincapié en aquello que tienen más confuso y ahondar en los asuntos que más les interesan. Para ello se han elaborado y realizado unos cuestionarios de preevaluación, que han permitido conocer qué conocimientos e ideas previas tenían. Estos mismos cuestionarios pasados al terminar cada tema a modo de posevaluación, han permitido obtener datos acerca de sus aprendizajes, nuevas ideas asimiladas y elaborar con ello, una escalera de aprendizaje de los estudiantes. Para dar sentido a estos cuestionarios con un valor cuantificable en la evaluación, se ha pedido a los estudiantes que los realicen bajo pseudónimo auto-asignado, de modo que no se sintiesen coaccionados o presionados. Con los datos resultantes hemos podido obtener la escalera de aprendizaje de cada estudiante con una gradación en 3 niveles alfabéticos, donde " $A$ " es el nivel más cualificado, " $B$ " el nivel medio, y " $C$ " el nivel manifiestamente mejorable. Los cuestionarios han sido 2. El primero para el tema 1; y el segundo para recoger contenidos del tema 2 y 3.

Se presenta los cuestionarios de pre y posevaluación siguientes que se realizan en este CIMA de 30 horas: 
Tabla 3. Cuestionario de pre/posevaluación de los temas 1 al 3

Cuestionario de pre y posevaluación del Tema 1

1. Qué es la cultura?

2. Qué valor tiene la diversidad cultural en la especie Homo Sapiens-Sapiens?

3. Podría existir una cultura universal y global?

4. Qué marcadores crees que identifican tu propia cultura?

5. Conoces algún grupo étnico? Qué marcadores identifican a ese grupo étnico?

6. Se producen actualmente procesos de genocidio, etnocidio y racismo? Pon un ejemplo de cada caso.

7. Qué es la interculturalidad y qué sentido tiene en un mundo globalizado?

8. Es lo mismo nación y nacionalismo? Qué relación tiene con la identidad étnica?.

Cuestionario de pre y posevaluación del Tema 2-3

1. Define comunidad imaginada. Con qué otro concepto lo relacionas?

2. Se puede considerar una génesis histórica para la difusión de los estados-nación a nivel mundial?. ¿dónde ubicarías su origen o matriz?

3. Cómo se significa la tradición en el imaginario nacional de sinitud?

4. Que es la niponidad y cómo la identificas?.

5. Es lo mismo grupo étnico que minoría etnolingüística? Por qué?. Qué sentido crees que tiene para la RPC?

6. Cuantas minorías étnicas tiene y cuántas reconoce China?

7. Cuantas minorías étnicas tiene y cuántas reconoce Japón?

8. Es lo mismo minoría étnica, minoría etnolingüística y diversidad cultural? Pon ejemplos aplicados en China y Japón.

En total, han realizado los cuestionarios 39 alumnos, por que el absentismo es importante en el Grado: hay muchos estudiantes que trabajan, ya que el titulo solo se oferta con mención en China y Japón en la Universidad de Sevilla (hay una especialidad en Corea en la Universidad e Málaga pues es un grado interuniversitario); los estudiantes que proceden de otras provincias, a veces realizan actividades laborales para poder mantenerse realizando sus estudios fuera de su localidad.

El de postevaluación del tema 1 lo realizaron 41 estudiantes; por tanto, 2 cuestionarios tuve que rehusarlos, 
porque sus autores no habían realizado el de preevaluación, de modo que no tenía datos medibles comparativos. Aparte de estas incidencias que han sido reguladas, se obtuvieron estos datos:

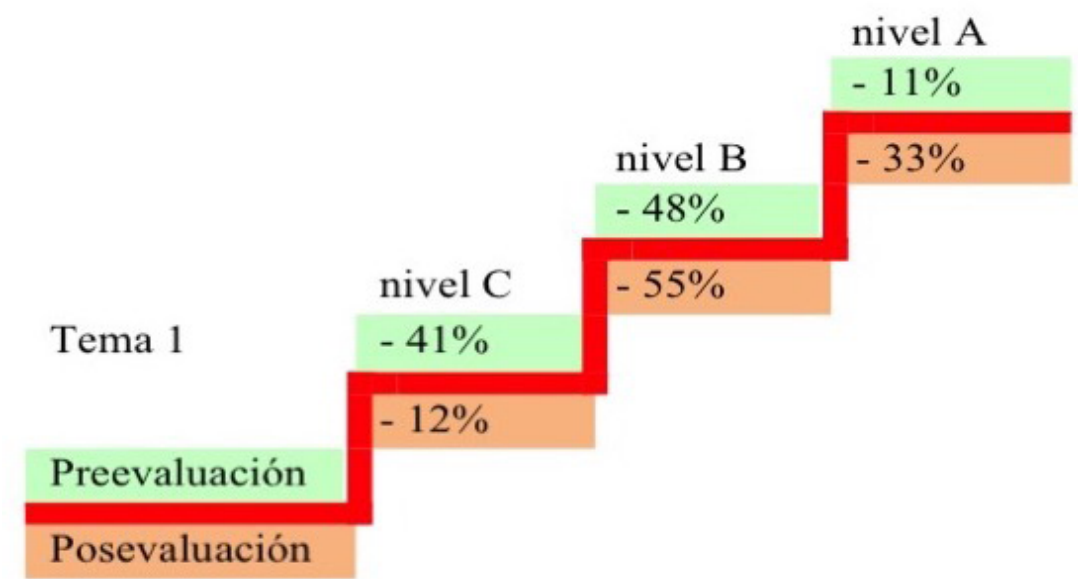

Figura 3. Escalera de aprendizaje Tema 1.



Figura 4. Escalera de aprendizaje Tema 2 y 3.

Cómo se puede observar en ambas escaleras los estudiantes manifiestamente han mejorado sus rendimientos desde el punto de vista de los aprendizajes, después de desarrollada la metodología docente diseñada. En el caso del Tema 1 se pasó de un $41 \%$ en el más bajo nivel a un 12\%, 
y se incrementó también en un considerable porcentaje el nivel más alto; en el caso del Tema 2 y 3 , de contenidos más complejos y que manejan contenidos transversales con otras asignaturas de historia moderna y contemporánea del título, inicialmente tenían un 52\% en el nivel más bajo, y se reduce a un 15\% después de impartidos y trabajados los contenidos con esta metodología.

\section{La evaluación de la docencia}

La evaluación de la docencia ha contado con varios instrumentos:

a) Observación entre pares sobre la comunicación en el aula en una sesión de 2 horas. Se han recogido las observaciones en la sesión de esta asignatura del día 16 de octubre por parte del profesor Jesús Sanbernardino. Por mi parte, yo realicé la misma observación en su asignatura de Historia Antigua que se imparte en primer curso (ver Jesús Sanbernardino, "La enseñanza y aprendizaje en Historia Antigua de Asia Oriental. Construyendo nuevas estrategias de innovación docente" en este mismo volumen).

En un diario de la sesión el prof. Sanbernardino recogió cada una de mis actividades y sus impresiones. Realizamos una reunión de intercambio y reflexión conjunta posterior acerca del experimento y sus resultados, a la luz que arrojaban nuestros propios diarios de las sesiones compartidas. El prof. Sanbernardino destacó la activación y la provocación con preguntas y re-preguntas con las que procuro promover la reflexión participativa en los estudiantes y mantenerlos activos en el proceso de comunicación de enseñanza-aprendizaje en el aula, así como el interés e implicación del alumnado en esta asignatura, y su capacidad de respuesta y el esfuerzo en hacer discursos elaborados sobre las problemáticas que se les plantea. 
También en su sesión, los estudiantes se caracterizan por este interés pro-activo ante las dinámicas que él realiza; esto facilita que nuestros esfuerzos de innovación sean gratamente percibidos y resulte interesante para nosotros continuar con estas metodologías.

b) He llevado un diario de las sesiones prácticas para evaluar la participación activa de los estudiantes y su respuestas ante los recursos utilizados en la enseñanza.

He podido constatar que hay un grupo de 6-7 estudiantes especialmente participativos que dan lugar a una dinámica activa general, en un grupo presencial que oscila entre los 30 y los 42 estudiantes. También es un dato de interés haber constatado que son especialmente pro-activos en las clases más participativas a partir de herramientas audiovisuales (documentales y films) que ante textos escritos (lecturas de artículos).

c) También he realizado, del mismo modo que venía haciendo en mis asignaturas del Grado de Antropología Social desde 2016, un cuestionario sobre la metodología docente empleada. Diseñado por mí y completamente distinto al oficial que se basa en ítems y cálculos muy estandarizados que poco me pueden orientar en la mejora en el aula. El cuestionario tiene 11 preguntas breves tipo test que grosso modo recogen información acerca del método, las praxis, la comunicación y el aprendizaje de contenidos conceptuales, actitudinales y procedimentales. Y tienen 2 apartados para que el estudiante puntúe la enseñanza y el trabajo de la profesora, y un apartado final especialmente interesante para que escriban libremente observaciones y recomendaciones de mejora. Estas observaciones de los estudiantes sirven para la reflexión posterior y la introducción de mejoras en otros diseños de CIMA posteriores. 
Tabla 4. Cuestionario de evaluación de la docencia

Cuestionario de evaluación de la docencia y la metodología

1. Los cuestionarios de pre y posevaluación Poco

te han ayudado en tu aprendizaje?

Bastante/suficiente

Mucho/muy bueno

2. Los recursos docentes para ayudarte en tu trabajo del proyecto-reto en coautoría han sido apropiados?

Poco

Bastante/suficiente

Mucho/muy bueno

3. Has hecho uso de las tutorías para solventar dudas de tu propio proyecto?

$\mathrm{Si}$

no

4. Las sesiones en tutoría para tu proyecto te han servido de orientación?

Poco

Bastante/suficiente

Mucho/muy bueno

5. Han sido útiles las tutorías colectivas en el aula sobre el proyecto-reto?

Poco

Bastante/suficiente

Mucho/muy bueno

6. Los materiales didácticos en la plataforma virtual de enseñanza han sido útiles?

Poco

Bastante/suficiente

Mucho/muy bueno

7. Los documentales en las clases prácticas han sido apropiados para los temas a tratar?

Poco

Bastante/suficiente

Mucho/muy bueno

8. Has aprendido a partir del modelo de explotación de fuentes documentales y lecturas a través de las fichas bibliográficas?

Poco

Bastante/suficiente

Mucho/muy bueno

9. Crees que las clases participativas te han permitido aprender y transmitir lo que sabes?

Poco

Bastante/suficiente

Mucho/muy bueno

Jornadas de Formación e Innovación Docente del Profesorado | № 2 (2019) Esta obra se distribuye con la licencia Creative Commons 
10. La profesora comunica bien los contenidos en el aula?

11. Crees que lo que has aprendido te servirá

para otras asignaturas del Grado?

\begin{abstract}
12. Evalúa la metodología de enseñanza que has recibido. De 1 a 10 siendo 5 aprobado.
\end{abstract}

Poco

Bastante/suficiente

Mucho/muy bueno

Poco

Bastante/suficiente

Mucho/muy bueno

13. Evalúa el trabajo docente de la profesora. De 1 a 10 siendo 5 aprobado

14. Dedica algo de tiempo a explicar cómo te habría gustado hacerlo a ti, y qué cosas cambiarías.

Realizaron el cuestionario 32 estudiantes presentes el último día del CIMA en el aula. Los resultados muestran que el porcentaje más alto aprecia el esfuerzo de la profesora por poner en juego otras metodologías docentes (92\%). También es generalizada la satisfacción por estar haciendo un tipo de proyecto con trabajo escrito cuyos aprendizajes pueden trasladar procedimentalmente a sus Trabajos Fin de Grado con el que culminan su titulación (95\%). Un porcentaje muy alto considera que gracias a esta asignatura adquieren conocimientos de "otros modos de ser asiático" o de "otras culturas asiáticas subalternas" (83\%), lo que es reflejo también del interés y la pertinencia de esta asignatura en el grado. Evalúan muy bien las metodologías participativas, con una nota media de sobresaliente (9,2 puntos); así como el trabajo y el esfuerzo de la profesora (9 puntos). Hay que tomar en cuenta que los estudiantes que hacen estas valoraciones han optado decididamente por la evaluación presencial, y han venido trabajando conmigo y con los compañeros en el aula. El absentismo supone el $50 \%$ del alumnado matriculado, y adoptan un método no presencial. Desconozco cuántos de ellos hacen la evaluación de la docencia en el sistema institucionalizado de la Universidad de Sevilla. Lo que he 
podido comprobar comparativamente en cursos anteriores es que suelo tener una buena valoración de mi docencia en esta asignatura de este título, mejor que en las que imparto en la titulación de Antropología Social. Y que los estudiantes presenciales de este grado responden muy bien a la innovación docente.

El apartado 14, me resulta de especial interés, porque a través de sus observaciones puedo introducir cambios el próximo curso: algunos estudiantes solicitan más recursos audiovisuales, debido a que no tienen ocasión de conocer la diversidad étnica en estos países. Como señala un estudiante que dice haber realizado una estancia internacional gracias a las becas de intercambio universitario que esta titulación tiene en la RPC y Japón, y que pueden disfrutar los estudiantes destacados en idiomas, el contexto de las estancias tampoco lo favorece. Ciertamente, es una tarea pendiente del titulo adquirir materiales educativos de este tipo para la formación universitaria. Esto me obliga a ser creativa ante esta deficiencia de cara al futuro, ya que los documentales mediáticos de acceso digital de las parrillas televisivas no responden a los contenidos de esta asignatura, y los de acceso libre en youtube a menudo no son útiles porque están en lenguas maternas no traducidas. También solicitan conferencias especializadas, confirmando la ausencia de estos temas en las conferencias que se organizan en el grado. Otra deficiencia que no he podido solucionar, por dificultad de acceso a recursos específicos de la titulación que posibiliten estas actividades.

\section{Reflexiones finales}

Aunque este el primer curso que realizo un ciclo de mejora docente en el Grado en Estudios de Asia Oriental, los resultados han sido muy positivos. Considero que ha sido acertado hacer hincapié en una metodología que potencie la adquisición de aprendizajes procedimentales que los estudiantes pueden transferir en su trabajos prácticos en otras asignaturas. Suplir la dificultad de partir 
del conocimiento del medio como generador de lo experimental por actividades centradas en aprender-haciendo, e incrementar el peso de las actividades y aprendizajes prácticos en la carga total del curso, hace que los estudiantes tengan una actitud proactiva en su aprendizaje y tomen las riendas de su propia formación. Creo que es muy útil y mucho más interesante trazar una urdimbre entre lo procedimental y lo teórico-conceptual a través del constructivismo educativo, activo y personal que los propios discentes pueden articular con sus habilidades tecnológicas bien orientadas (Rodríguez y Ramírez, 2014).

La evaluación de la docencia, y de los mapas mentales de los estudiantes, nos permite tener un juicio fundamentado del impacto o de los logros de nuestras actividades (Rivero y Porlán, 2017:74). Actividad que me ha resultado muy interesante en esta asignatura y que voy a establecer el próximo curso también.

Estas metodologías no sólo hacen sujetos participativos a los estudiantes, sino que los contenidos llegan a través de las propias fuentes científicas que tienen que aprender a encontrar, gestionar e interpretar. Es la lógica que Finkel promueve con "dar clases con la boca cerrada" (Finkel, 2008:45) y "dejar que hablen los libros" (Finkel, 2008:56). Es lo que he tratado de orientar a través de la selección de autores de referencia de teoría general y enfocados en Asia Oriental, promoviendo con preguntas la reflexión aplicada hacia las culturas nacionales y étnicas que estudian.

También considero que es muy necesario profundizar con el prof. Jesús Sanbernardino en la observación entre pares como una actividad interesante de innovación docente, que permite "verse" como docente a través de otro compañero. Compartir las dificultades que cada uno de nosotros tiene en su espacio propio, y compartir herramientas de trabajo o diseños, permite canalizar dinámicas de cambio y mejora. 


\section{Referencias bibliográficas}

Finkel, D. (2008) Dar clase con la boca cerrada. Universitat de València.

Rivero, A. y Porlán, R. (2017). La evaluación en la enseñanza universitaria. En R. Porlán (Coord.), Enseñanza universitaria. Cómo mejorarla (73-91). Madrid: Ediciones Morata. Rodríguez García, A. B. y Ramírez López, L. J. (2014) Aprender haciendo-investigar reflexionando: caso de estudio paralelo en Colombia y Chile (53-63) Revista Academia y Virtualidad, 7 (2).

Jornadas de Formación e Innovación Docente del Profesorado | № 2 (2019) Esta obra se distribuye con la licencia Creative Commons 